\title{
SUZAN: Modelo Fuzzy para Classificação Espectral de Dados de Supernovas Ia
}

\author{
Luís Ricardo Arantes Filho ${ }^{1}$ \\ Instituto Nacional de Pesquisas Espaciais \\ Lamartine Nogueira Frutuoso Guimarães ${ }^{2}$ \\ Instituto de Estudos Avançados \\ Reinaldo Roberto Rosa ${ }^{3}$ \\ Instituto Nacional de Pesquisas Espaciais \\ Francisca Joamila Brito do Nascimento ${ }^{4}$ \\ Instituto Nacional de Pesquisas Espaciais
}

\begin{abstract}
Resumo. Supernovas são cataclismos cósmicos. Estas explosões representam o ciclo final de evolução das estrelas. A classificação do fenômeno é complexa e está condicionada à perícia de um astrônomo que identifica as peculiaridades do espectro óptico e das linhas espectrais. Desta forma, este trabalho apresenta SUZAN (Sistema fUZzy Avaliador de superNovas) um método de classificação de supernovas utilizando o paradigma da Lógica Fuzzy, simulando o especialista humano no momento da análise espectral e da classificação espectral. SUZAN avalia um grande número de espectros obtendo seu melhor desempenho para supernovas Ia em sua fase de luminosidade máxima.
\end{abstract}

Palavras-chave. Supernovas, Fuzzy, classificação espectral, supernovas Ia.

\section{Introdução}

Supernovas (SN) são caracterizadas por explosões extremas no cosmos. Estas explosões derivam-se do ciclo final de evolução de uma estrela massiva ou de reações termonucleares em estrelas anãs brancas e podem gerar quantidades de energia e luminosidade equiparadas ao brilho total de uma galáxia. A importância da classificação deste fenômeno está na constatação das explosões de Supernovas do tipo Ia (SNIa) como sendo uma classe de explosões com luminosidade máxima pouco variável de objeto a objeto, o que as torna réguas padronizáveis para medidas de distâncias cosmológicas. Este importante passo científico rendeu o prêmio Nobel de Física de 2011 aos astrofísicos Saul Perlmutter, Brian P. Schmidt e Adam G. Riess $[9,10]$ que constataram a expansão acelerada do universo através da observação de supernovas distantes.

\footnotetext{
${ }^{1}$ luisricardoengcomp@gmail.com

${ }^{2}$ lamar.guima@gmail.com

${ }^{3}$ rrrosa.inpe@gmail.com

${ }^{4}$ francisca.nascimento@inpe.br
} 
Esta importante descoberta foi derivada da análise das curvas de luz (magnitude em função do tempo) das SNIa, esta análise só é possível num período de 40 a 60 dias após a explosão de cada SN. Em contrapartida, é possível identificar as SNIa pelo espectro (fluxo de radiação em função do comprimento de onda), que é observado assim que determinado o alvo pelos telescópios e espectrógrafos $[3,4,8]$. A análise das linhas espectrais de emissão e absorção (picos e vales) permite identificar a composição química do objeto, as SNIa são ricas em Silício (Si) e Enxofre $(\mathrm{S})$ e não apresentam traços de Hidrogênio $(\mathrm{H})$ ou Hélio (He) $[1,2,4]$. Neste artigo descrevemos um sistema baseado em Lógica Fuzzy modelado pela inferência de Takagi-Sugeno [11] para classificação automática de um grande volume de dados espectrais de SNIa. Os dados utilizados nesta pesquisa pertencem ao acervo The CfA Supernova Archive [2,5] que contém espectros de diversos tipos de SN, principalmente SNIa. Apresentamos o sistema SUZAN (Sistema fUZzy Avaliador de superNovas) que executa a análise espectral de SN de forma semelhante ao astrônomo especialista, sendo capaz de avaliar e classificar as SNIa com maior precisão quando o espectro está no período próximo ao brilho máximo da explosão.

A justificativa para a adoção do paradigma da lógica fuzzy está no desenvolvimento de um sistema capaz de simular o conhecimento especialista sem a necessidade de um treinamento de dados prévio para identificação de padrões, utilizando portanto, toda a amostra de dados na validação do modelo. O desenvolvimento da SUZAN foi pensado como uma alternativa ao CIntIa (Classificador Inteligente de supernovas do Tipo Ia) proposto por [8] e posteriormente aprimorado por [3] (CINTIA2) que utiliza o conceito de redes neurais artificiais e por isso, necessita de uma grande quantidade de exemplos para relacionar corretamente os padrões de SN. Estes sistemas necessitam que os modelos sejam treinados com uma proporção de $80 \%$ dos dados originais, validando os resultados de classificação em $20 \%$ dos dados. O sistema SUZAN é estabelecido em um cenário em que a análise de SN demanda agilidade, e é por isso que a avaliação espectral é construída já que os espectros são um dos primeiros tipos de dados em que é possível realizar uma análise de componentes químicos em SN.

\section{Modelagem Fuzzy no sistema de Inferência de Takagi- Sugeno}

O modelo Fuzzy permite a graduação na pertinência de elementos à determinada classe. A função que define o grau de pertencimento de um determinado elemento em um conjunto fuzzy é denominada função de pertencimento e é definida segundo o critério de um especialista ou empiricamente. Em relação à classificação quando são utilizadas várias entradas de dados (padrões), muitas funções de pertencimento devem ser utilizadas para melhor representar as regras que definem se um padrão pertence a determinado conjunto ou não [11]. O modelo de Inferência de Takagi-Sugeno consiste em um tipo de inferência capaz de descrever funções de forma aproximada e é muito utilizado em aplicações de controle. As etapas para o desenvolvimento do modelo são: a seleção de entradas; a modelagem das regras e a escolha das funções de pertencimento; a ativação das regras; e a inferência. Desta forma, este modelo permite que se tenha uma relação linear de entrada 
e saída (input-output), As funções de associação de saída neste modelo, são lineares ou constantes [11]. As regras deste modelo são descritas de acordo com a definição (2.1).

Definição 2.1. Se $k 1$ é B1 e k2 é B2 ... kn é Bn, Então $y=f i(k 1, k 2, \ldots, k n)$.

Em que B1,..., Bn são os conjuntos fuzzy dos antecedentes enquanto que o consequente é uma função das variáveis de entrada $\mathrm{k} 1, \mathrm{k} 2, \ldots, \mathrm{kn}$ e $\mathrm{n}$ é o número de regras que são ativadas. As funções representam os polinômios gerados pela inferência do modelo vista em (1). A saída gerada por este sistema de inferência é polinomial [11].

$$
y=\frac{\sum_{i=1}^{n} w_{i} f_{i}(k 1, k 2, \ldots, k n)}{\sum_{i=1}^{n} w_{i}} .
$$

A ativação das regras é dada por um operador de implicação e é definido pela variável $w_{i}$. Este operador é definido para interpretar as informações das regras se-então interpretando os conectivos E, OU e CASO CONTRÁRIO. O operador de implicação é definido em $(2.2)$.

Definição 2.2. $\phi\left[\mu_{A}(x), \mu_{B}(y)\right] \equiv \phi\left[\mu_{A}(x) \wedge \mu_{B}(y)\right]$.

No modelo de Takagi-Sugeno de ordem zero as regras são definidas conforme (2.3).

Definição 2.3. Se k1 é B1 e k2 é B2 ... kn é Bn, Então $y=S$.

Tem-se um modelo de Takagi-Sugeno de ordem zero se o polinômio é uma constante. Neste ponto, S é definida como um consequente, ou seja, uma variável de saída que é dependente das variáveis de entrada $k_{n}$. Esta variável assume valores constantes e pode ser representada também por termos linguísticos e seus respectivos valores. A saída gerada é um polinômio de ordem zero. Quando a saída é uma constante as funções de associação são definidas como singletons [11], e os métodos de implicação e agregação das regras são fixos. A equação (2) descreve a inferência para o modelo de Takagi-Sugeno de ordem zero.

$$
y=\frac{\sum_{i=1}^{n} w_{i} S_{i}}{\sum_{i=1}^{n} w_{i}} .
$$

O método de inferência de Takagi-Sugeno de ordem zero aplicado neste trabalho foi escolhido pela forma como as saídas são geradas na avaliação fuzzy. Este modelo permite que o custo computacional para realizar as operações seja reduzido, pois os consequentes são polinômios de grau zero.

\subsection{Modelagem da SUZAN}

A modelagem fuzzy do sistema SUZAN ocorre com a correta identificação das linhas espectrais do espectro de SN conforme a normalização de dados feita em [3]. Esta normalização compreende uma filtragem dos dados espectrais e o ajuste do redshift para avaliar as linhas espectrais do objeto em repouso. Este ajuste é feito para que as linhas espectrais de elementos de supernovas estejam ajustadas o mais próximo das linhas espectrais de elementos que são obtidas no laboratório. A partir da correta localização das linhas 
espectrais de SN são abstraídos dois parâmetros de características: as larguras $(W)$ e intensidades de pico $\left(p_{k}\right)$, como visto na Fig. 1 . Na Fig.1 os valores de $F_{\lambda}$ e $F_{c}$ correspondem respectivamente aos valores de fluxo do espectro da linha de absorção e do envoltório para delimitação da área da linha espectral. As variáveis $\lambda_{1}$ e $\lambda_{2}$ correspondem aos limites da linha espectral e recebem os valores do comprimento de onda. Ainda na Fig. $1, h$ é a altura baseada nos fluxos do espectro, ou seja, $h=\left(F_{c}-F_{\lambda}\right)$. Desta forma é possível calcular os valores de $W$ e $p k$, o calculo é feito de forma relativa para as linhas de emissão.

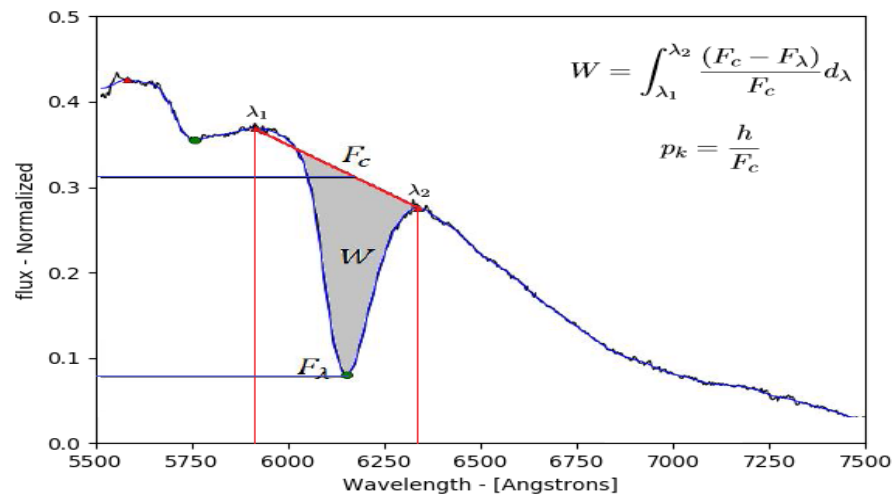

Figura 1: Cálculo das características $W$ e $p_{k}$ relacionadas às linhas espectrais.

O algoritmo fuzzy (regras fuzzy) define se uma linha espectral tem características estreitas, medianas ou largas. Quanto maior for o valor de $W$ e mais intenso for $p_{k}$ então a linha se enquadra como sendo "larga", gradativamente quando os valores são pequenos as características assumem o valor de mediana, estreita, declínio e nulas (valores baixos de $p_{k}$ e $W$ ). As linhas avaliadas pela SUZAN como largas, medianas e estreitas são utilizadas na análise de elementos químicos. Assim são definidas duas outras variáveis fuzzy (Distância e Elementos fuzzy) para avaliar e definir os elementos $\mathrm{Si}, \mathrm{S}$, He e H para cada linha espectral selecionada anteriormente. A Fig.2 (a,b e c) ilustra como são modelados os parâmetros $W, p_{k}$ e de Distância para funções de pertencimento. A modelagem Fuzzy (construção de funções de pertencimento, termos linguísticos e regras fuzzy) seguiu um critério para definir o quanto uma linha espectral é "larga" e com "grande intensidade"e o quanto uma linha é "estreita" e com "baixa intensidade". Para definir o universo de discurso de cada função foi definida a largura e a intensidade média de cada linha nas regiões do Si, S, H e He. A Fig. 2 (d) mostra todos os espectros da base de dados de SNIa e o padrão médio (linha azul) em diferentes períodos (fases espectrais definidas em relação ao brilho máximo da explosão de SN, por exemplo, a definição Fase: +4 refere-se a 4 dias após o pico da explosão de SN, Fase: -4 refere-se a 4 dias antes do pico da explosão).

A variável Distância define a proximidade (comprimento de onda) da linha selecionada para o valor de comprimento de onda dos elementos que caracterizam as SN. Esta variável foi criada, pois mesmo com o ajuste de redshift os valores de elementos dos espectros são imprecisos em relação aos valores de elementos de laboratório. A variável Elementos fuzzy foi definida como uma região de comprimento de onda que representa um dos elementos que aparecem nos espectros de $\mathrm{SN}(\mathrm{Si}, \mathrm{S}, \mathrm{He}$ e $\mathrm{H})$, desta forma quanto mais próxima a 


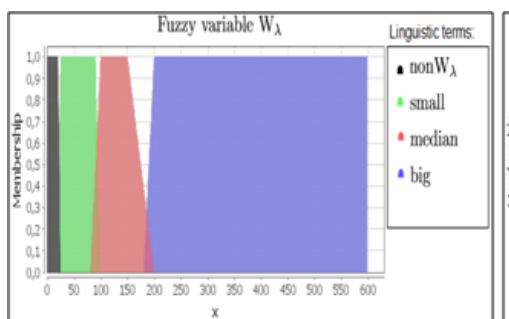

(a)

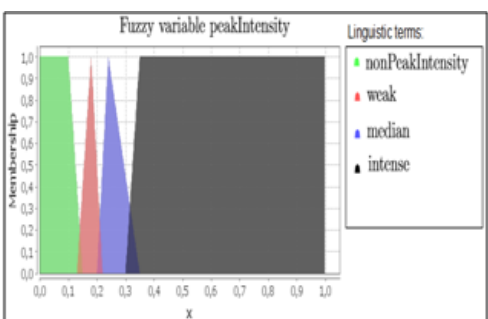

(b)

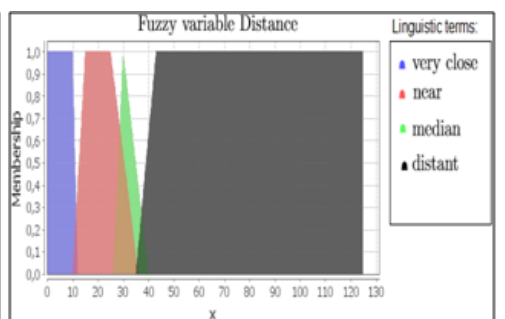

(c)
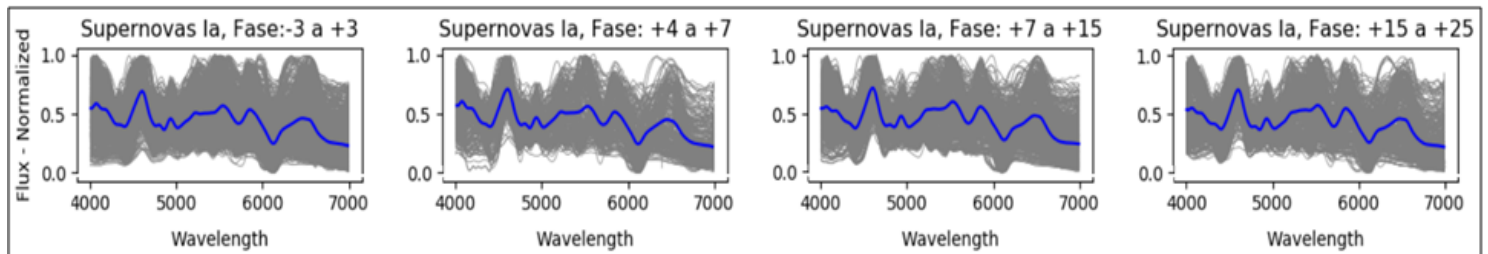

(d)

Figura 2: Modelagem das Funções de Pertencimento. (a) Corresponde a modelagem da variável fuzzy $W$, (b) corresponde a modelagem da variável fuzzy $p_{k}$ e (c) corresponde à variável fuzzy de Distância. Em (d) é ilustrado o padrão médio dos espectros de SNIa em diferentes fases de observação, que foi utilizado como critério para modelagem das variáveis fuzzy.

linha avaliada está desta região maior é o grau de pertencimento a este elemento. A Fig. 3 indica como estes elementos definem a classificação dos tipos clássicos de SN. Quando SUZAN identifica a presença Si e o $\mathrm{S}$ no espectro e a ausência do $\mathrm{H}$ e do He, então o espectro é classificado como SNIa.
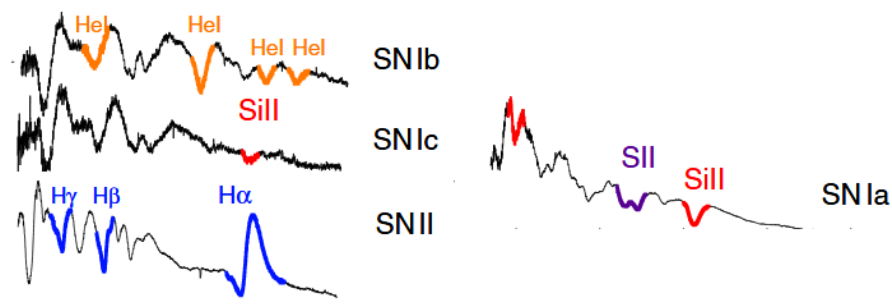

Figura 3: Esquema de classificação de elementos em SN [7].

\section{Resultados}

Foram avaliados os dados de SNIa do acervo The CfA Supernova Archive [5]. A Tab. 1 descreve os dados brutos que foram submetidos a análise da SUZAN, principais referências e características temporais dos espectros, bem como, o desempenho da classificação para cada base.

A análise espectral de SNIa pode ser feita com precisão no intervalo de 5 dias em relação ao brilho máximo da explosão, ou seja, quando a curva de luz da SN atinge seu 
Tabela 1: Dados espectrais de SN e desempenho da SUZAN na classificação das SNIa.

\begin{tabular}{ccccc}
\hline Principal referência & Tipo de SN & Qtde. Espectros & Fase Espectral (dias) & Acertos $(\%)$ \\
\hline$[1]$ & SNIa & 47 & $-2.5 \mathrm{a}+2.5$ & 100 \\
{$[2]$} & SNIa & 2603 & $-15 \mathrm{a}+611$ & 71,92 \\
{$[6]$} & SNIa & 432 & $-13 \mathrm{a}+243$ & 76,15 \\
Total & - & 3082 & - & - \\
\hline
\end{tabular}

ponto máximo são contados 2.5 dias antes do máximo e 2.5 dias depois do máximo (-2.5 dias a +2.5 dias). Neste período as características do Si e do S são mais evidentes, como apontam $[1,4]$ e é neste período em que os astrônomos especialistas executam a classificação de maneira visual. Desta forma, para verificar a eficiência da SUZAN em avaliar as SNIa apenas neste período foram correlacionados espectros que mantinham suas características principais de elementos dentro do período de tempo de -2.5 a +2.5 dias conforme a Tab. 2 .

Tabela 2: Avaliação da SUZAN para SNIa próximas ao brilho máximo.

\begin{tabular}{ccccc}
\hline Principal referência & Tipo de SN & Qtde. Espectros & Qtde de SN & Acertos $(\%)$ \\
\hline$[1]$ & SNIa & 47 & 26 & 100 \\
{$[2]$} & SNIa & 634 & 462 & 96,21 \\
{$[6]$} & SNIa & 91 & 32 & 97,80 \\
Total & - & 772 & 520 & 95,85 \\
\hline
\end{tabular}

\section{Considerações sobre a Abordagem}

Neste trabalho destaca-se a análise de SNIa no período em que as SNIa atingem seu brilho máximo, identificando aproximadamente $96 \%$ (conforme a Tab. 2) dos espectros de SNIa nesta fase. SUZAN possui uma imprecisão na classificação de SNIa quando as fases espectrais estão muito distantes da fase de brilho máximo da SN (conforme a Tab. 1), nestes longos períodos as características morfológicas dos espectros variam, pois é visualizado o espectro da nuvem de gás da explosão da estrela que se dissipa no decorrer do tempo. Estas variações nas características dos espectros indicam a presença de outros elementos, como o Ferro (Fe), tornado a classificação de SNIa ainda mais complexa. As funções de pertencimento e regras fuzzy da SUZAN foram modeladas para SNIa próximas ao período de -2.5 a +2.5 dias em relação à luminosidade máxima, pois é neste período em que o astrônomo observa as características dos elementos $(\mathrm{Si}, \mathrm{S}, \mathrm{H}, \mathrm{He})$ e executa a classificação. Apresentamos um modelo fuzzy capaz de modelar o conhecimento de um astrônomo e executar de maneira automática a classificação espectral de SNIa. A correta classificação e identificação de SNIa por sistemas automáticos propicia contribuição referente a estudos cosmológicos que avaliam a expansão acelerada do universo, haja visto que sistemas classificadores de SN como a SUZAN, proporcionam uma análise precisa sobre 
um grande volume de dados em um curto período de tempo algo difícil para especialistas humanos.

\section{Agradecimentos}

Agradecemos ao INPE e ao IEAV pelo apoio à pesquisa, bem como, ao CNPq e à CAPES.

\section{Referências}

[1] S. Blondin, K. S. Mandel, and R. P. Kirshner. Do spectra improve distance measurements of type ia supernovae?,Astronomy \& Astrophysics, 526(A81), 2011. DOI: https://doi.org/10.1051/0004-6361/201015792.

[2] S. Blondin, et al. The spectroscopic diversity of type ia supernovae, The Astronomical Journal, 143(5): 126, 2012. URL: http://stacks.iop.org/1538-3881/143/i=5/a=1.

[3] F. J. B. do Nascimento, L. R. Arantes Filho, and L. N. F. Guimarães. Intelligent Classification of Supernovae Using Artificial Neural Networks.Inteligencia Artificial, 22(63), 39-60, 2019. DOI: https://doi.org/10.4114/intartif.vol22iss63pp39-60.

[4] A. V. Filippenko. Optical spectra of supernovae. Annual Review of Astronomy and Astrophysics, v.35, n. 1, p. 309-355, 1997.

[5] CfA. The CfA Supernova Archive. Harvard-Smithsonian Center for Astrophysics, 60 Garden Street, Cambridge, MA 02138, 2018. URL: https://www.cfa.harvard.edu/supernova/SNarchive.html.

[6] T. Matheson, et al. Optical spectroscopy of Type Ia supernovae. The Astronomical Journal, v. 135, n.4, p. 1598, 2008.

[7] M. Modjaz, et al. Optical spectra of 73 stripped envelope core-collapse supernovae. The Astronomical Journal, v. 147, n. 5, p. 99, 2014.

[8] M. Módolo. Classificação Automática de Supernovas Usando Redes Neurais Artificiais. Tese (Doutorado em Computação Aplicada) — Instituto Nacional de Pesquisas Espaciais (INPE), São José dos Campos, 2016. URL: http://mtcm21b.sid.inpe.br/col/sid.inpe.br/mtc-m21b/2016/04.20.20.44/doc/publicacao.pdf.

[9] S. Perlmutter, et al. Measurements of omega and lambda from 42 high-redshift supernovae. The Astrophysical Journal, v. 517, n. 2, p. 565, 1999.

[10] A.G. Riess, et al. Observational evidence from supernovae for an accelerating universe and a cosmological constant. The Astronomical Journal, v. 116, n. 3, p. 1009, 1998.

[11] T. Takagi, M. Sugeno. Fuzzy identification of systems and its applications to modeling and control. IEEE transactions on systems, man, and cybernetics, n. 1, p. 116-132, 1985 . 